\title{
Metal bead crystals for easy heating by direct current
}

Bert Voigtländer, Vasily Cherepanov, Christa Elsaesser, and Udo Linke

Citation: Review of Scientific Instruments 79, 033911 (2008);

View online: https://doi.org/10.1063/1.2894302

View Table of Contents: http://aip.scitation.org/toc/rsi/79/3

Published by the American Institute of Physics

\section{Articles you may be interested in}

Preparation of bead metal single crystals by electron beam heating

Journal of Vacuum Science \& Technology A: Vacuum, Surfaces, and Films 23, 1535 (2005); 10.1116/1.2101793

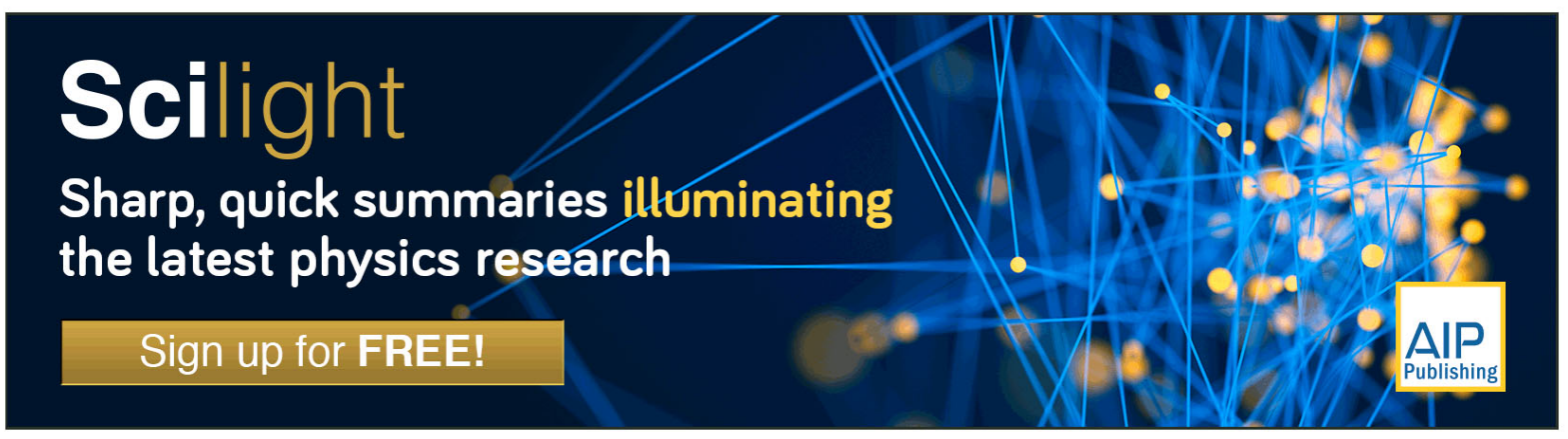




\title{
Metal bead crystals for easy heating by direct current
}

\author{
Bert Voigtländer, ${ }^{\text {a) }}$ Vasily Cherepanov, Christa Elsaesser, and Udo Linke \\ Institut für Bio- und Nanosysteme IBN-3 and Center of Nanoelectronic Systems for Information Technology \\ (cni), Forschungszentrum Jülich, 52425 Jülich, Germany
}

(Received 24 December 2007; accepted 16 February 2008; published online 31 March 2008)

\begin{abstract}
The preparation of metal bead crystals with two wires attached to the crystal is described. These crystals allow for a very easy and efficient method to heat metal single crystals by direct current heating through the connecting wires of the bead crystal. This heating of the bead crystal is sufficient to clean metal surfaces such as the surfaces of $\mathrm{Pt}$ and $\mathrm{Au}$ as confirmed by Auger spectroscopy and scanning tunneling microscopy (STM). There is no need for any ion sputtering which is conventionally used to clean metal single crystal surfaces. The bead crystals with two leads fabricated from a wide range metals and metal alloys such as $\mathrm{Cu}, \mathrm{Mo}, \mathrm{Ru}, \mathrm{Rh}, \mathrm{Pd}, \mathrm{Ag}, \mathrm{Ta}, \mathrm{W}, \mathrm{Re}$, Ir, Pt, Au, PtPd, PtRh, AuAg, and PtIr can be used as general purpose metal substrates for surface science studies and other applications. Additionally, these bead crystals can be used to reshape STM tips by indentation of the tip into the soft metal in order to recover atomic resolution imaging on hard substrates. () 2008 American Institute of Physics. [DOI: 10.1063/1.2894302]
\end{abstract}

\section{INTRODUCTION}

Metal single crystals used in surface science studies in ultrahigh vacuum (UHV) have usually a relatively large diameter of $10 \mathrm{~mm}$ or more and have to be cleaned after inserting into UHV. This cleaning is commonly performed by repeated cycles of ion bombardment and subsequent annealing. Such a heating system is usually an electron beam heater or some resistive heating element electrically isolated from the metal single crystal. These heating systems have the disadvantage that they require a lot of power. From this point of view, a direct current heating would be advantageous. With this heating technique, ideally only the crystal is heated resistively and no heater is required. Unfortunately, metal crystals have a low resistance and therefore a very high current is required for direct current heating making this approach impractical due to very thick leads which would be required.

Here, we describe a method to fabricate small $(2-4 \mathrm{~mm}$ diameter) bead crystals with two wires attached to the bead single crystal. These two-lead bead crystals allow for an extremely simple method to heat the surface of bead crystal just by direct current heating through the leads. Since in direct current heating, only the crystal and no additional heater is required, the desired temperature can be reached with a minimal power. Also, due to the small size of the bead crystals of typically $2 \mathrm{~mm}$, they are specifically suited for applications in which only a small space is available for the sample. Such applications include scanning probe applications and specific applications such as tip enhanced Raman spectroscopy. The heating procedure is very much simplified and can be performed at a minimum pressure due to the low power dissipated. As we will show here, in some cases (spe-

\footnotetext{
a) Author to whom correspondence should be addressed. Electronic mail:
} b.voigtlaender@fz-juelich.de. cifically for Pt), only sample heating is required to clean the bead crystal surface and no sputtering is necessary to clean the sample.

The sample cleaning by direct current heating of a bead crystal allows also another important application. In STM, it turned out that when a hard substrate (such as silicon) is scanned by a tungsten tip, the atomic resolution is lost after some time and it is difficult to recover atomic resolution once it is lost. On the other hand, on metal substrates which are softer than tungsten a reshaping of the tip after atomic resolution has been lost (by indentation of the tip into the soft metal substrate during STM operation) is an every day practice and the tungsten STM tips have virtually never to be changed when tunneling on soft metal samples. If the STM is operated in UHV, the installation of a new tip is a quite time consuming procedure which includes tip etching, introduction into UHV, cleaning of the tip to remove the oxide layer, and finally mounting to the STM. An alternative procedure would be to use a soft metal sample to reshape the tip and then return to the hard sample with a recovered tip. If the metal sample is not cleaned, a lot of contaminations reside at the surface which will be transferred to the tip apex during indentation of the tip. These contaminations will also degrade the imaging quality of the STM tip. Therefore, it is advantageous to clean the sample of the soft metal before a new tip apex is pulled by indentation. With the easy possibility to clean a $\operatorname{Pt}(111)$ bead crystal with two wires by direct current heating, a metal single crystal can be used for STM tip shaping also in systems used for semiconductor studies where no dedicated equipment for the cleaning of metal samples is available.

The two lead bead crystals can be fabricated from a wide variety of metals and metal alloys ${ }^{1}$ such as $\mathrm{Cu}, \mathrm{Mo}, \mathrm{Ru}, \mathrm{Rh}$, $\mathrm{Pd}, \mathrm{Ag}, \mathrm{Ta}, \mathrm{W}, \mathrm{Re}, \mathrm{Ir}, \mathrm{Pt}, \mathrm{Au}, \mathrm{PtPd}, \mathrm{PtRh}, \mathrm{AuAg}$, and PtIr and are commercially available. ${ }^{2}$ They can be used as small, ex- 


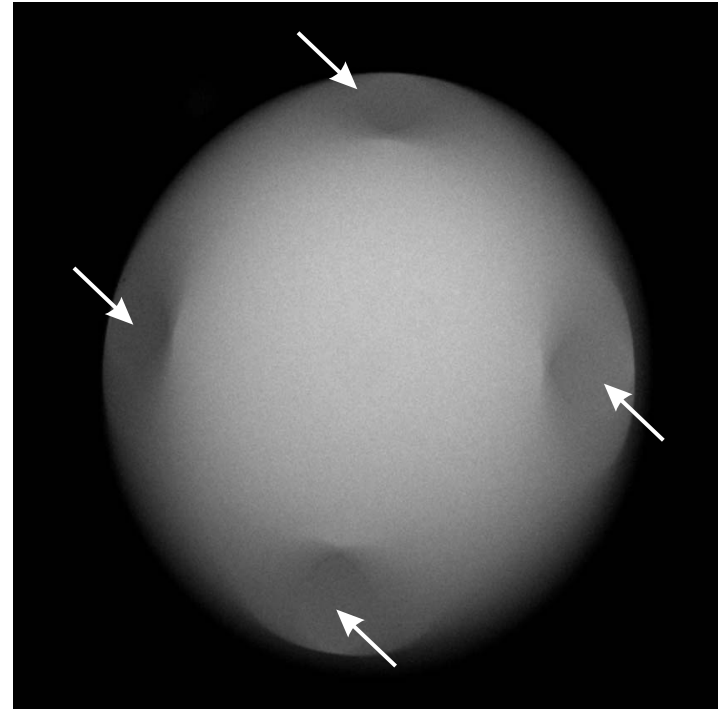

FIG. 1. SEM image of a platinum bead crystal (diameter $\sim 2.5 \mathrm{~mm}$ ). Low index (111) facets on the bead crystal are indicated by arrows.

tremely simple to heat and to clean single crystal substrates for studies in surface science, thin film growth, electrochemistry, and other fields.

\section{FABRICATION AND CLEANING OF A TWO-LEAD BEAD CRYSTAL}

Bead crystals with one wire are made by melting the end of a polycrystalline noble metal wire (typically $0.1-1 \mathrm{~mm}$ diameter). The end of the wire is molten using a fine gas flame. When the gas flame is moved along the wire axis, a liquid metal droplet (or bead) is formed at the end of the wire. When the liquid metal bead is slowly cooled, the bead crystallizes with one (or sometimes several) of the crystal grains in the solid wire serving as nucleus for the crystal structure of the solidified bead crystal. ${ }^{3}$ After some time of melting and recrystallization of the bead crystal, the metal bead solidifies from only one nucleus and a bead single crystal has formed as shown in the scanning electron microscopy (SEM) image shown in Fig. 1. The facets marked in Fig. 1 arise due to facets on the equilibrium crystal shape of a platinum crystal. ${ }^{4}$ The above described method for the fabrication of a bead crystal is a well known procedure. ${ }^{3}$ Single crystal metal bead crystals are predominantly used in electrochemistry. ${ }^{5-10}$ Recently, the preparation of bead single crystals was extended to electron beam heating in vacuum. ${ }^{1}$ This allows fabricating bead crystals from a larger variety of materials than just the noble metals, previously accessible. The heating in vacuum prevents the oxidation of reactive metals occurring under ambient conditions and allows the fabrication of bead crystals from a wide variety of metals and metal alloys.

Here, we describe a method to fabricate metal bead crystals with two wires [Fig. 2(a)] (actually, also the fabrication of bead crystals with three or more wires is possible). The two leads can be used to run a current through the crystal, which is an efficient way to heat the crystal. For the fabrication of the two-wire bead crystal two metal wires (diameter of $0.1-0.5 \mathrm{~mm}$ ) are twisted around each other at one end and
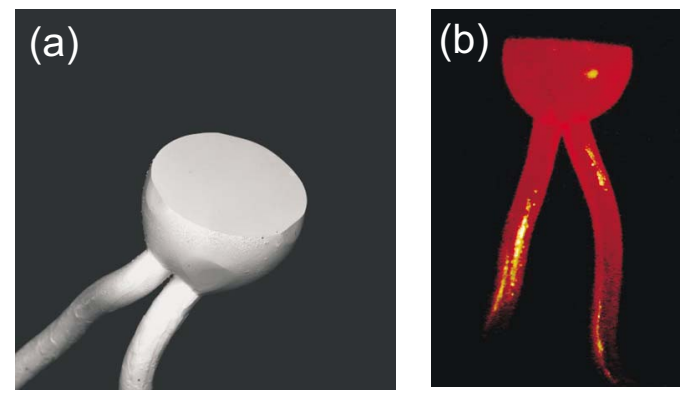

FIG. 2. (Color online) Platinum bead crystal (diameter $\sim 2.5 \mathrm{~mm}$ ) with two wires. The top surface is oriented along (111) and polished. The two wires are used to heat the sample by direct current heating. (b) Homogeneous heating to red heat is shown in this image.

molten at the twisted end of the wires. The melting of the wires can be performed by a gas flame under ambient conditions but most favorably the melting is performed by electron beam heating under vacuum conditions. ${ }^{1}$ The vacuum environment prevents oxidation and the formation of gas inclusions. As a result, a molten metal drop (diameter $\sim 2 \mathrm{~mm}$ ) forms, similar to the case of the one wire bead. After some repeated tries of melting and solidification, one single crystal nucleus extends from one wire over the bead toward the second wire, the metal wire solidifies to one single crystal bead. For most materials, the bead crystals exhibit characteristic $\left(\begin{array}{lll}1 & 1 & 1\end{array}\right)$ facets due to their low surface energy ${ }^{11}$ (see Fig. 1). These facets are used for the crystallographic orientation of the crystals. In other cases, $\mathrm{x}$-ray diffraction is used for the orientation of the crystals. The bead crystals are gently etched with aqua regia to remove accumulated impurities and to inspect the grain distribution. To obtain larger areas of low index orientation surfaces, the crystal is cut along one of the low index facets to obtain a low crystal surface [for instance, (111)]. Finally, the low index crystal surface is polished. For polishing, the crystals are mounted on a customdesigned holder and preoriented with a $\mathrm{He}-\mathrm{Ne}$ laser. Subsequently, the bead was mechanically polished down to a hemispherical shape to increase the geometric area of the desired surface orientation. Polishing was performed using a $1200 \mathrm{SiC}$ wax/aqueous suspension followed by polishing with diamond paste of various grain sizes and a final polishing step with $0.03 \mu \mathrm{m} \mathrm{Al}_{2} \mathrm{O}_{3}$ in a slightly alkaline glycerine/ water mixture on a soft felt. A finished Pt bead crystal (diameter of $2 \mathrm{~mm}$ ) with two leads (diameter of $0.5 \mathrm{~mm}$ ) and a polished (111) facet is shown in Fig. 2(a). These bead crystals manufactured from various metals such as $\mathrm{Cu}, \mathrm{Mo}, \mathrm{Pd}$, Ag, Ta, Pt, Au, PtPd, PtRh, AuAg, and PtIr and are also commercially available. ${ }^{2}$ In the case of $\mathrm{Ru}, \mathrm{Rh}, \mathrm{W}, \mathrm{Re}$, and Ir, bead crystals can be fabricated even with two wires, however, the connecting wires are very brittle.

The heating procedure for such a small bead crystal in UHV is extremely simple. Using the two wires as leads, the crystal is heated by running a current through the leads. Figure 2(b) shows a Pt crystal which is heated very homogeneously to red heat by direct current heating. It is no problem to heat the crystal also to higher temperatures. For instance, a temperature of $1000^{\circ} \mathrm{C}$ has been reached for a Pt bead crystal for heating currents as low as 4 A. In Fig. 3(a), the 

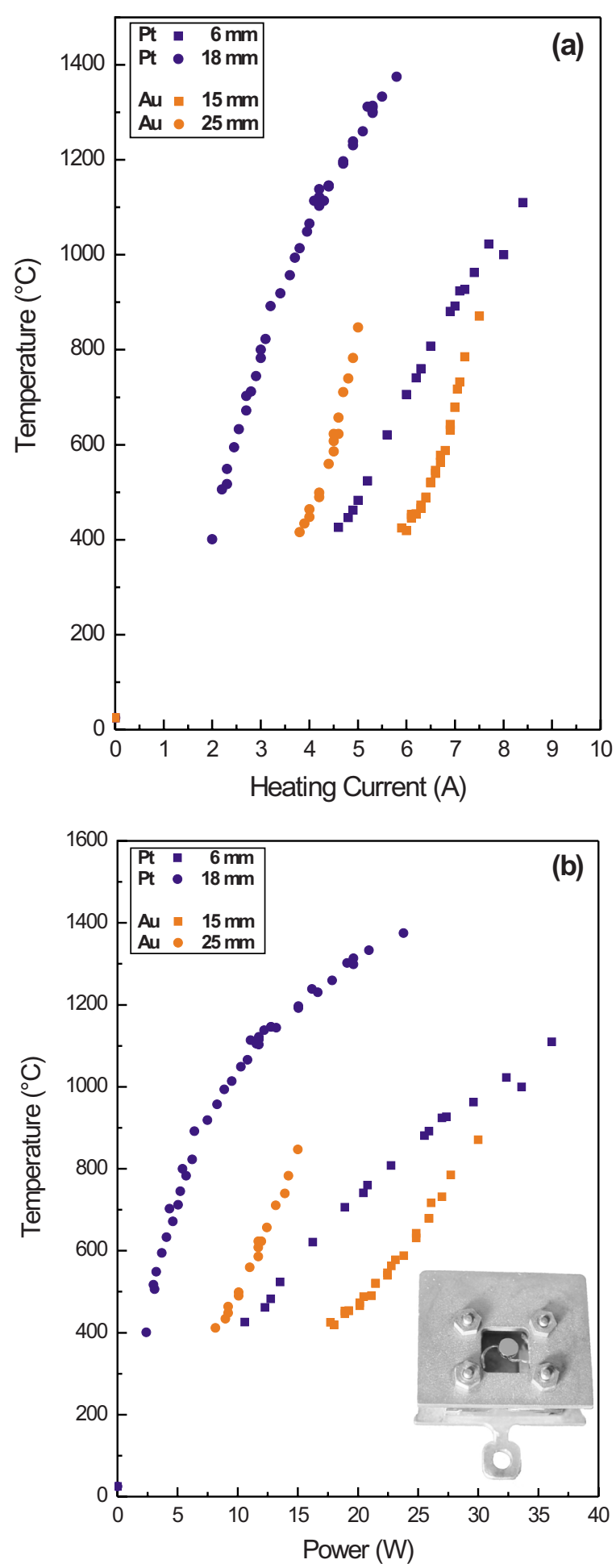

FIG. 3. (Color online) (a) Temperature of the bead crystal as function of the heating current for Pt and Au bead crystals with different wire lengths. (b) Temperature of the bead crystal as function of the heating power. In the inset in (b), a bead crystal mounted in an Omicron ${ }^{\circledR}$ sample holder is shown.

temperature measured by an infrared pyrometer is plotted against the heating current for $\mathrm{Au}$ and $\mathrm{Pt}$ bead crystals of a diameter of $2 \mathrm{~mm}$. In the case of the Au bead crystal, the maximum temperature is $\sim 870^{\circ} \mathrm{C}$ because this temperature is already less than $200^{\circ} \mathrm{C}$ from the melting point of $\mathrm{Au}$ $\left(1063^{\circ} \mathrm{C}\right)$. Due to the lower specific electrical resistivity of $\mathrm{Au}$, the heating currents are larger than for the case of $\mathrm{Pt}$ bead crystals. As the bead crystal is heated by the power dissipated by direct current heating through the leads (wire diameter of $0.3 \mathrm{~mm}$ ), the temperature reached for a certain current depends strongly on the length of the leads. In Fig. 3(a), the temperature versus heating current is shown for two different wire lengths of $6 \mathrm{~mm}$, and $18 \mathrm{~mm}$ in the case of Pt. The heating is most efficiently for the long wire length. On the other hand, the long wire length has the disadvantage that the mounting is not so stable and vibrations have been observed in STM experiments. If, however, short wire length of less than $18 \mathrm{~mm}$ are used the vibration amplitude in STM measurements was well below $0.1 \AA$. The total power dissipated to reach a certain temperature is shown in Fig. 3(b). The large increase of the heating power for shorter wire length shows that the main part of the heating power is dissipated by thermal conduction toward the sample holder. The heating characteristic given in Fig. 3 was obtained for our specific sample mounting shown in the inset of Fig. 3(b). Besides, on the wire length, the power needed to reach a certain temperature depends also on the details of the mounting and the sample holder since this defines how much heat is dissipated toward the sample holder. In our current setup, the bead crystal wires are just clamped to the sample holder [inset in Fig. 3(b)]. In an optimized setup, one would take more care to reduce the heat flow from the wire to the sample holder. By this, the heating power can be reduced considerably.

One advantage of the direct current heating is that the sample is the hottest part and a pyrometer can be used for easy direct temperature measurement. Using other heating methods has the disadvantage that the heater is the hottest part and the sample is often (much) cooler than the heater. This has the disadvantage that temperature measurement using an infrared pyrometer is almost impossible because the measured temperature is influenced strongly by the heater temperature or by the glowing filament in the case of the electron beam heating. Another advantage of the direct current heating is that, in this case, there is no contact of any hot heating element directly to the sample. This way, any possible contaminations of the sample from a heating element are prevented.

The low power dissipation during the direct current heating is also beneficial in order to heat the crystal at low pressure during heating. A low power during heating also means that the parts surrounding the sample are less heated and this to a low pressure during sample heating and to less contaminants desorbed from the sample holder during sample heating. We have tried to clean the surface of the Pt bead crystals by heating alone, i.e., without ion etching of the sample. The cleanness of the Pt bead crystal was checked by Auger spectroscopy using a cylindrical mirror analyzer detector. The only contamination detected before heating of the $\mathrm{Pt}(111)$ bead crystal was carbon with a measured ratio $\mathrm{C}_{272} / \mathrm{Pt}_{64}$ of 0.8 . We successively heated the $\operatorname{Pt}(111)$ bead crystal for about $10 \mathrm{~min}$ and recorded an Auger spectrum afterward. The $\mathrm{C}_{272} / \mathrm{Pt}_{64}$ peak ratio as function of annealing temperature is shown in Fig. 4. For temperatures exceeding $700^{\circ} \mathrm{C}$, a continuous decrease of the carbon signal was observed. After heating for $30 \mathrm{~min}$ to $1105^{\circ} \mathrm{C}$, the carbon signal is 


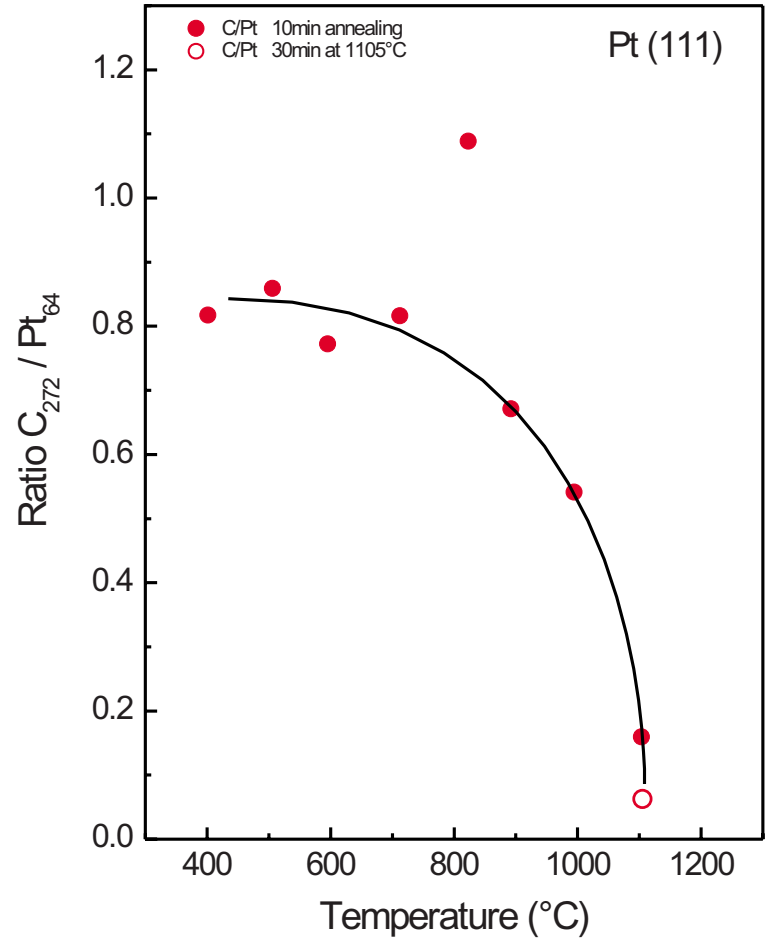

FIG. 4. (Color online) Auger electron spectroscopy of the $\mathrm{C}_{272} / \mathrm{Pt}_{64}$ peak ratio as function of annealing temperature for a $\mathrm{Pt}(111)$ bead crystal. For temperatures exceeding $700^{\circ} \mathrm{C}$, a continuous decrease of the carbon signal was observed. After heating for $30 \mathrm{~min}$ to $1105^{\circ} \mathrm{C}$, the carbon signal is dropping below the background level.

dropping below the background level. The Auger spectrum does not show any other peaks than the ones expected for a clean Pt crystal. Therefore, we found that heating the Pt(111) bead crystal at $1100^{\circ} \mathrm{C}$ in UHV at a pressure of less than $1 \times 10^{-9} \mathrm{mbar}$ is sufficient to clean the crystal surface from contaminations. One reason why only heating is an effective method for cleaning of the $\operatorname{Pt}(111)$ bead crystal is the fact that the fabrication of the bead crystals uses clean starting materials ( $\mathrm{Pt}$ wires of $99.9 \%$ purity) and uses subsequently a very clean manufacturing procedure (melting in a $\mathrm{H}$ flame or electron beam heating in vacuum). During the normal manufacturing method of metal single crystals, electrical discharge machining (EDM) is used to cut the crystals from the single crystal rod. This EDM is a very dirty machining technique which pumps a lot of carbon and other contaminants into the metal which have to be removed afterward. With the bead crystals, we use much cleaner materials and processes to start with resulting in a much lower level of contaminations from the beginning and retain this much cleaner material during the fabrication of the bead crystal. The bead crystal is purified even more by the zone melting process used in the process of the bead crystal fabrication.

The crystal surface is checked for residual contaminations also with the help of STM. STM images of the surface of the Pt(111) bead crystal (Fig. 5) show miscut steps of one to three atomic layers (step height of $0.23 \mathrm{~nm}$ ). The terraces and the step edges, which are the usual locations for residual contaminations, do not show any indication of contamination. Since this extremely quick and easy way of cleaning of a single crystal surface is beneficial, we tried to transfer this

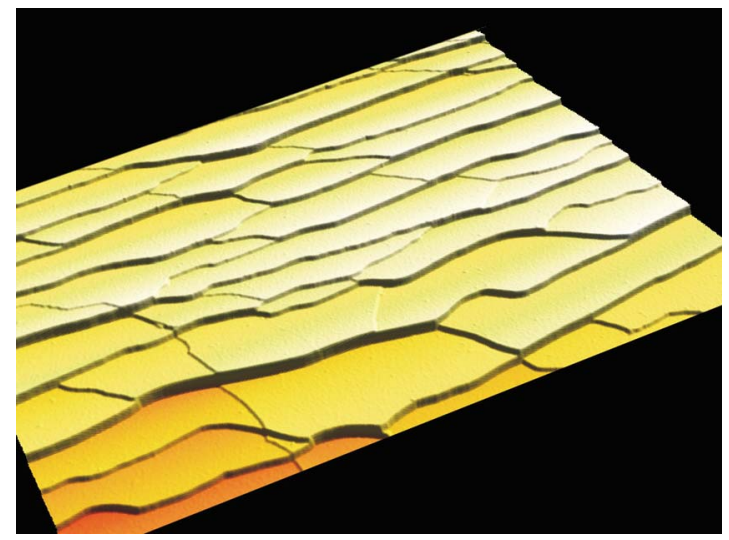

FIG. 5. (Color online) Rendered STM image of the Pt(111) surface of the bead crystal after direct current heating. Clean terraces as well as clean steps of monoatomic height and double or triple height are visible (image size of $530 \times 260 \mathrm{~nm}^{2}$ ).

method also to other materials. For the case of $\mathrm{Au}$, the cleaning procedure turned out to be much more difficult. Also, for the case of $\mathrm{Au}$, the carbon Auger signal decreased for annealing temperatures higher than $700^{\circ} \mathrm{C}$. However, to remove carbon completely, a temperature close to the melting point of $\mathrm{Au}\left(1063^{\circ} \mathrm{C}\right)$ is required. Since the two connecting heating wires have a somewhat higher temperature than the bead crystal itself, most often, these wires molt before the bead crystal was clean from carbon. In one case, the connecting wires did not melt and a clean bead crystal free from carbon contaminations was obtained, as confirmed by Auger spectroscopy. A STM image of this clean Au(111) bead crystal sample showing the typical herringbone reconstruction is shown in Fig. 6.

\section{RESHAPING OF THE STM TIP BY CONTROLLED INDENTATION INTO A BEAD CRYSTAL}

In this section, we describe, as an application of the usage of bead crystals, how a cleaned bead crystal is used to

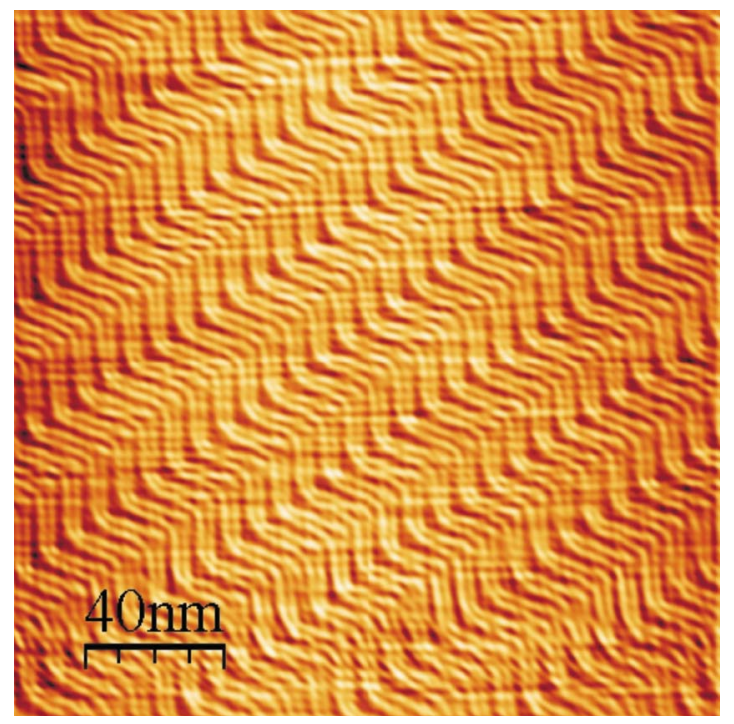

FIG. 6. (Color online) STM image of the herringbone reconstruction on a $\mathrm{Au}$ bead crystal with (111) surface orientation. The surface was cleaned by thermal treatment only without sputter cleaning the surface. 

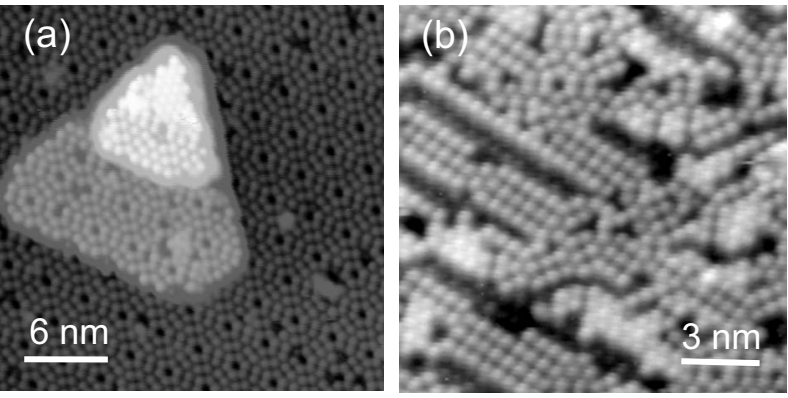

FIG. 7. (a) Atomic resolution STM image of a Si(111) surface taken with a reshaped tip. The $(7 \times 7)$ reconstruction is visible, as well as the reconstruction on top of an island demonstrating the recovery of atomic resolution after reshaping. (b) Surface of a $0.6 \mathrm{~nm}$ thick Si layer on Ge(111) shows, that also nonperiodic surfaces are imaged with atomic resolution.

reshape a STM tip which does not provide atomic resolution imaging on a Si sample any more. A cleaned Pt bead crystal is introduced as sample in the STM and the STM tip is reshaped by controlled indentation of the tip into the platinum sample. The detailed procedure of the indentation of the tip is as follows. Tip and sample are approached to the tunneling condition. The tip is retracted from tunneling by applying a voltage to the $z$ piezo. Then, the STM feedback loop is switched off. After setting the sample voltage to $10-12 \mathrm{~V}$, the STM tip is approached toward the sample by changing the voltage applied to the $z$ piezo until the STM tip touches the sample surface (i.e., a current is measured). The tip is further moved toward the sample until a current of $\sim 10 \mathrm{~mA}$ through the tip is measured by a multimeter which is connected to the tip instead of the STM preamplifier. This current is limited by a $1 \mathrm{k} \Omega$ resistor. Subsequently, the tip is slowly retracted by changing the $z$-piezo voltage. The duration of this process until the current drops to zero should be about $1 \mathrm{~min}$. After retraction of the tip, the preamplifier can be reconnected and the feedback loop can be switched on again. The tip quality can be checked immediately by scanning the Pt bead sample. If the steps on the sample are imaged sharp, imaging of the hard material with the reshaped tip results usually in atomically resolved images. If the steps on the Pt sample cannot be resolved sharply, the reshaping procedure should be performed once more. In Fig. 7(a), an atomically resolved image taken on a $\mathrm{Si}(111)$ surface after reshaping of the STM tip is shown. The $7 \times 7$ reconstruction is visible on the substrate as well as on the $\mathrm{Si}$ island. In Fig. 7(b), an example of a more disordered surface is shown [ $\mathrm{Si}$ on $\mathrm{Ge}(111)]$. Also, this more disordered structure is resolved atomically after reshaping of the tip.

We think that the Pt sample melts locally due to the current which is confined to a very small region at the apex. If the tip is retracted subsequently, a new tip apex is pulled from the local Pt melt and the tip apex is coated by the soft platinum. One important property of such a Pt coated tip is that atomic resolution can be obtained for a much longer time compared to the original tungsten tip. Also, after an (unintentional) tip-sample contact (tip crash), the atomic resolution is usually not lost, unlike with the original tungsten tip. The indentation of the Pt coated tip into the hard material ( $\mathrm{Si}$, in our case) leads to the formation of a new microtip which provides atomic resolution imaging. Moreover, if the atomic resolution is lost during scanning of the hard material with a Pt covered (reshaped) tip, the resolution will be restored after some time of scanning. This beneficial behavior is specific to the reshaped tips and is not observed on tungsten tips. Generally, a Pt coated tip maintains atomic resolution for a much longer time, than the original tungsten tip.

To provide an even easier switching between the semiconductor sample and the Pt bead crystal used for tip shaping, we constructed a double sample holder hosting a semiconductor sample as well as a Pt bead crystal. Both samples can be heated independently by direct current heating and cross contamination is prevented by molybdenum shield between both samples. Using this sample holder, one can switch back and forth between the semiconductor sample and the bead crystal for tip reshaping within a couple of minutes.

\section{CONCLUSIONS}

The preparation of metal bead crystals with two wires attached to the crystal has been described. These crystals allow for a very easy and efficient method to heat metal single crystals by direct current heating through the connecting wires of the bead crystal. This heating of the bead crystal is sufficient to clean metal surfaces such as the (111) surface of Pt as confirmed by Auger spectroscopy and STM. The use of time consuming procedures such as sputtering with noble gas ions is not necessary. The bead crystals with two leads fabricated from a wide range of metals and metal alloys such as $\mathrm{Cu}, \mathrm{Mo}, \mathrm{Ru}, \mathrm{Rh}, \mathrm{Pd}, \mathrm{Ag}, \mathrm{Ta}, \mathrm{W}, \mathrm{Re}, \mathrm{Ir}, \mathrm{Pt}, \mathrm{Au}, \mathrm{PtPd}, \mathrm{PtRh}$, $\mathrm{AuAg}$, and PtIr can be used as general purpose low index metal surface substrates for studies in surface science, thin film growth, electrochemistry, and other fields. Additionally, these bead crystals can be used to reshape STM tips by indentation of the tip into the soft metal in order to recover atomic resolution imaging on hard substrates on which atomic resolution is usually lost quickly.

\section{ACKNOWLEDGMENTS}

We would like to acknowledge the help of J. Myslivecek in constructing the double sample holder.

${ }^{1}$ B. Voigtlander, U. Linke, H. Stollwerk, and J. Brona, J. Vac. Sci. Technol. A 23, 1535 (2005).

${ }^{2}$ http://www.icryst.com

${ }^{3}$ J. Clavilier, R. Faure, G. Guinet, and R. Durand, J. Electroanal. Chem. Interfacial Electrochem. 107, 205 (1980).

${ }^{4}$ T. Wang, C. Lee, and L. D. Schmidt, Surf. Sci. 163, 181 (1985).

${ }^{5}$ K. Sashikata, N. Furuya, and K. Itaya, J. Vac. Sci. Technol. B 9, 457 (1991).

${ }^{6}$ K. Sashikata, Y. Matsui, K. Itaya, and M. P. Soriaga, J. Phys. Chem. 100, 20027 (1996).

${ }^{7}$ L.-J. Wan, M. Hara, J. Inukai, and K. Itaya, J. Phys. Chem. B 103, 6978 (1999).

${ }^{8}$ N. Fuuya, M. Ichinose, and M. Shibata, Phys. Chem. Chem. Phys. 3, 3255 (2001).

${ }^{9}$ L. A. Kibler and D. M. Kolb, Z. Phys. Chem. 217, 1265 (2003).

${ }^{10}$ M. Hara, U. Linke, and Th. Wandlowski, Electrochim. Acta 52, 5733 (2007).

${ }^{11}$ S. Surnev, K. Arenhold, P. Coenen, B. Voigtländer, H. P. Bonzel, and P. J. Wynblatt, J. Vac. Sci. Technol. A 16, 1059 (1998). 